\title{
BMJ Open Workflow interruptions and stress atwork: a mixed-methods study among physicians and nurses of a multidisciplinary emergency department
}

\author{
Matthias Weigl, ${ }^{1}$ Joana Beck, ${ }^{1}$ Markus Wehler, ${ }^{2}$ Anna Schneider ${ }^{1}$
}

To cite: Weigl M, Beck J, Wehler M, et al. Workflow interruptions and stress atwork: a mixed-methods study among physicians and nurses of a multidisciplinary emergency department. BMJ Open 2017;7:e019074. doi:10.1136/ bmjopen-2017-019074

- Prepublication history for this paper is available online. To view these files, please visit the journal online (http://dx.doi. org/10.1136/bmjopen-2017019074).

MW and AS contributed equally.

Received 10 August 2017 Revised 14 November 2017 Accepted 15 November 2017

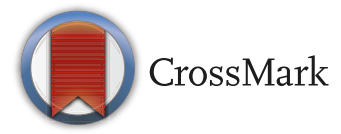

1 Institute and Outpatient Clinic for Occupational, Social and Environmental Medicine, Ludwig-Maximilians-University Munich, Munich, Germany ${ }^{2}$ Department of Emergency Medicine and Medicine IV, Klinikum Augsburg, Augsburg, Germany

Correspondence to Dr Matthias Weigl; matthias.weig|@med.Imu.de

\section{ABSTRACT}

Objectives Dealing with multiple workflow interruptions is a major challenge in emergency department (ED) work. This study aimed to establish a taxonomy of workflow interruptions that takes into account the content and purpose of interruptive communication. It further aimed to identify associations of workflow interruptions with ED professionals' work stress.

Design Combined data from expert observation sessions and concomitant self-evaluations of ED providers.

Setting ED of an academic community hospital in Germany.

Participants Multidisciplinary sample of ED physicians and nurses. 77 matched observation sessions of interruptions and self-evaluations of work stress were obtained on 20 randomly selected days.

Outcome measures ED professionals' stress evaluations were based on standardised measures. ED workload data on patient load, patient acuity and staffing were included as control variables in regression analyses.

Results Overall mean rate was 7.51 interruptions/ hour. Interruptions were most frequently caused by ED colleagues of another profession (27.1\%; mean interruptions/hour rate: 2.04 ), by ED colleagues of the same profession $(24.1 \% ; 1.81)$ and by telephone/beeper (21\%; 1.57). Concerning the contents of interruption events, interruptions most frequently occurred referring to a parallel case under care $(30.3 \%, 2.07)$, concerning the current case $(19.1 \% ; 1.28)$, or related to coordination activities $(18.2 \%, 1.24)$. Regression analyses revealed that interruptive communication related to parallel cases significantly increased ED providers' stress levels $(\beta=0.24$, $P=0.03)$. This association remained significant after controlling for ED workload.

Discussion Interruptions that refer to parallel cases under care were associated with increased stress among ED physicians and nurses. Our approach to distinguish between sources and contents of interruptions contributes to an improved understanding of potential benefits and risks of workflow interruptions in ED work environments. Despite some limitations, our findings add to future research on the implications of interruptions for effective and safe patient care and work in complex and dynamic care environments.
Strengths and limitations of this study

- Application of structured expert observation sessions ensures reliable identification of emergency department (ED) providers' workflow interruptions.

- Combination of observational data and provider ratings adds valuable information on ED staff experiences of interruptions.

- This study introduces an empirical quantification of interruption contents and interruptive communication in ED care settings.

- Study findings should be confirmed in multi-centre investigations

\section{INTRODUCTION}

Emergency departments (EDs) are complex and challenging work environments with significant demands for care providers and inherent risks for quality of patient care. ${ }^{1-3}$ Human factors and ergonomics contribute to the investigation and establishment of safe and efficient ED work. ${ }^{14}$ Although EDs are 'interrupt driven' care environments, ${ }^{56}$ the nature and impact of interruptions on provider stress and ED care is yet not well understood. ${ }^{12478}$

We define workflow interruptions as an intrusion of an unexpected task or communication event, causing a discontinuation of the current task and an observable task switch behaviour. ${ }^{9}$ Thus, interruptions suspend clinician's attention from the focal task, requiring a shift of attention and task switching. ${ }^{29} \mathrm{ED}$ physicians and nurses are frequently engaged in interruption-laden processes with 5.1 up to 15.5 interruptions/hour. ${ }^{2}{ }^{10-14}$ In EDs, interruptions tend to occur more frequently than in other clinical settings. ${ }^{210}$ Moreover, in the course of dealing with interruptions, ED physicians fail to return to their initial task up to $20 \%$ of the time or they compensate through task short cuts. ${ }^{15}$ 
Likewise, interruptions affect length of interventions in the $\mathrm{ED}^{16}$ and are associated with lower patient satisfaction. ${ }^{17}$

Current research predominantly addresses potential negative influence of workflow interruptions. ${ }^{71819}$ Workflow interruptions may mitigate clinicians' situational awareness and mental focus, increase fatigue, stress or frustration. ${ }^{20}$ Highly interruptive work environments may contribute to failures, errors and quality losses. ${ }^{4}$ In contrast and concerning potential beneficial effects, in an attempt to accomplish fast and efficient patient care in the ED, interruptions might be inherent to complex ED work. ${ }^{346}$ Thus workflow interruptions are purposeful to ensure fast and safe delivery of care or to foster immediate communication, task completion and information transfer in the ED. ${ }^{612-14} 19$

A previous review on interruptions in ED work called for further investigations that address proximal and distal outcomes of interruptions. ${ }^{7}$ Moreover, research is needed that elaborates potential adverse effects of interruptions as well as positive outcomes such that interruptions provide additional value to $\mathrm{ED}$ providers or organisational outcomes. ${ }^{71421}$ Therefore approaches that comprehend the complexity of interruptions in ED work are needed. ${ }^{714}$ We therefore sought to expand previous research that mostly focused on the sources of interruptions by proposing a newly developed classification of interruptions in ED work that accounts for the sources and contents of interruption events. ${ }^{714}$ By drawing on a mixed-methods approach with ED professionals, our study aimed to:

1. identify interruption sources and contents of interruption events in ED work

2. determine associations between interruptions and ED professionals' work stress.

\section{METHODS}

\section{Design and study setting}

This mixed-methods study combined expert observation sessions on workflow interruptions and concomitant self-assessments by ED professionals concerning perceived disruptiveness and work stress. It was conducted in the ED of a major metropolitan academic medical centre with 84000 yearly visits. In regard to size, patient census, work organisation, staffing and technological provisions, this interdisciplinary ED is one of the largest EDs in Germany. The study ED is staffed with physicians from trauma surgery, internal medicine and other specialties on 24 hours duty. The ED consists of three treatment areas according to patient's chief complaints and condition: examination and treatment rooms for (1) internal as well as (2) patients with trauma and (3) one observation and clinical decision unit.

Staff received written and verbal information prior to data collection. Participation was voluntary and written consent was obtained. This study was part of a research programme on ED professionals' work conditions and care quality.

\section{Sample and procedure}

Trained observers shadowed ED professionals in $90 \mathrm{~min}$ sessions on 20 days. Eligible professionals were ED nurses and physicians undergoing specialty training or with completed specialty degree who worked in ED care on the respective 20 days of data collection. Overall, 80 observation sessions of ED professionals' workflow were scheduled. Observation sessions were randomly assigned to ED treatment areas and professions.

\section{Observational and self-report measures}

Observation sessions included sources and contents of workflow interruptions (see table 1). For identification of (1) sources, an established observation tool with eight different categories was used. ${ }^{62}$ For (2) contents, we developed a taxonomy that distinguishes between seven different content categories of interruptive communication (see table 1). The first four categories draw on previous attempts to differentiate between case-related and non-case-related interruption events. ${ }^{23}$ Additionally, we included a category that accounts for interruptions that foster time and comfort aspects of patient care. ${ }^{14}$

\section{ED professionals' self-reports on interruptions and work stress}

Immediately after each observation session, the observed professional was asked to fill out a short self-assessment survey concerning the following outcomes:

\section{Perceived interruptions}

Three questions examined the perceived frequency, usefulness and disruptiveness of interruptions: (1) 'How often were you interrupted during your work?' (scale range from 0 'very few times' to 10 'very frequently'); (2) 'Were interruptions useful for your work (eg, to work more efficiently, to mitigate errors?' (0 'not useful', 10 'very useful'); (3) 'Were interruptions disruptive for your work?' (0 'not disruptive', 10 'very disruptive').

\section{Work stress}

An established scale that quantifies the cognitive, emotional and physical aspects of work stress was used. STAI-6 scale has been frequently applied in healthcare professionals. ${ }^{24}$ It consists of 6 statements with adjectives, for example, describing feeling calm (reversely coded), tense or upset. The answer scale ranges from 1 'no, not at all' to 4 'yes, completely'. The scale showed high reliability: Cronbach's alpha $=0.82$.

\section{Additional information}

Additional ED workload information was coded for each observation session: (1) treatment area, and (2) profession. Additionally, data was obtained on ED patient load (number of patients registered), ED staffing (number of physicians and nurses) and number of high acuity patients (share of patients with ESI levels 1 and 2).

\section{Reliability testing}

First, non-systematic observation sessions were carried out to test the applicability of the tool and to discuss 
Table 1 Sources and contents of emergency department (ED) professionals' workflow interruptions (observation tool)
Category
Example

(1) Sources of interruptions (interruptions caused...)

$\begin{array}{lll}1 & \text { By patients } & \text { For example, patient asks charting nurse for expected waiting time } \\ 2 & \begin{array}{l}\text { By ED colleagues of the same } \\ \text { profession }\end{array} & \begin{array}{l}\text { For example, charting nurse is interrupted by another ED nurse who asks for } \\ \text { missing chart }\end{array}\end{array}$

3 By ED colleagues of another profession

For example, charting nurse is interrupted by ED physician who informs her about the arrival of a new patient

\begin{tabular}{|lll}
4 & By telephone/beeper & For example, ED physician receives a telephone call during patient examination \\
\hline 5 & By patient's relatives & For example, woman asks charting nurse about her mother's diagnostic results \\
\hline 6 & $\begin{array}{l}\text { By any other person or professional } \\
\text { not working in the ED }\end{array}$ & For example, police, ambulance personnel, or external service personnel \\
\hline
\end{tabular}

7 By technical malfunctions or missing For example, computer screen is frozen, ECG runs out of battery supplies

8 By information impediments or $\quad$ For example, necessary information for task completion is missing, patient chart problems is unavailable

(2) Contents of interruptions (interruption event...)

1 Related to current case Interruption provides information on current case of observed ED professional; for example, nurse informs physician about an external call from patient's husband.

2 Related to parallel case

Interruption provides information on another patient that is currently under treatment by observed ED professional but not in immediate care; for example, physician informs nurse that her patient in parallel room needs assistance with getting dressed, or nurse assistant informs nurse that her patient in the parallel room is going to be transferred to the operating room (OR).

\section{Related to completed cases \\ 4 Related to a new case (time-critical)}

$5 \quad$ Related to coordination activities

6 Related to patient comfort
Interruption provides information on a patient whose ED care has been completed or who is not under current care by observed ED professional; for example, nurse informs physicians about yesterday's referral of a trauma patient.

Interruption provides information on a new critical patient who is not physically present in the ED yet; for example, beeper sets off with announcement of arrival of a new trauma patient.

Interruption is not related to a specific patient but rather to the coordination of ED workflow or collaboration; that is, nurse informs physician about her absence for a break.

Interruption refers to maintaining or improving patient experience and comfort in general; for example, relatives ask nurse where to find restrooms.

7 Others Interruption events that cannot be classified.

inconsistencies. Afterwards, observation sessions with two trained observers were conducted on site to test tool's reliability. Finally, 14 pairwise observation sessions were carried out (sum: 15 hours, $23 \mathrm{~min}$; range: 0:36-1:30). To avoid temporal misclassification, observational data was divided into $1 \mathrm{~min}$ phases. For each, both observer classification of interruption sources and contents were matched respectively. A total of 274 interruptions sources were coded with a resulting Kappa coefficient of 0.56 . Concerning content of interruptions, a total of 269 scores were obtained (for five events, rates missed a classification). The resulting Kappa coefficient was 0.50 . For both domains, inter-rater agreement was established.

\section{Data analyses}

Missing self-assessments of work stress were registered for three observation sessions, in two instances high patient load prevented observed professionals to respond immediately afterwards. In three other observation sessions, observed ED professionals finished their shift prior to the planned session end (mainly due to shift changes) and other ED professionals followed within the same role. Here, self-evaluations of both observed ED professionals within the same observation session were aggregated for mean evaluation. Observational data and self-report data were matched for each of the observation sessions. Sum and mean values were computed. For each observation session, interruption rates were calculated respectively, that is, session's count of interruption events divided by its length. Analyses of variance were performed to explore group differences between professions and ED treatment areas. We then computed multivariate linear regression analyses between observed rates of interruptions and ED 


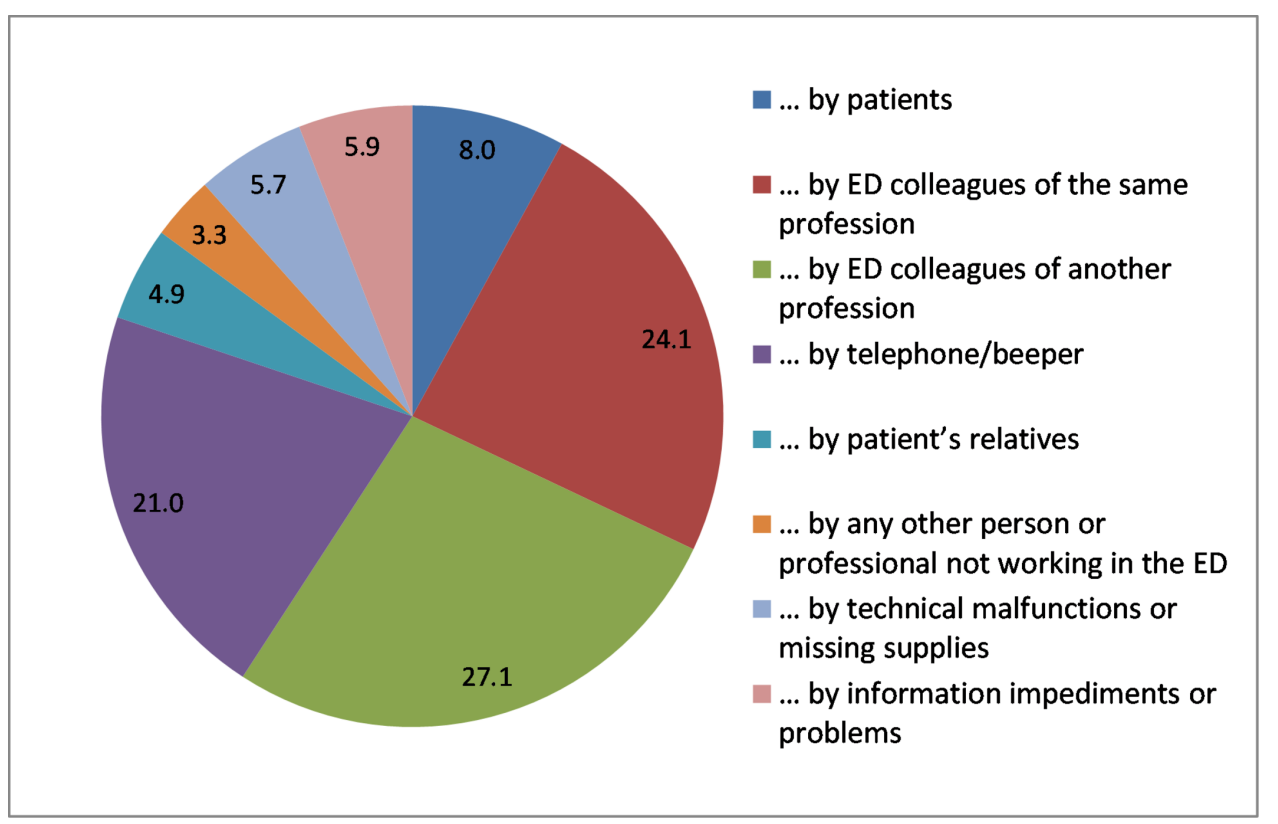

Figure 1 Sources of emergency department (ED) providers' workflow interruptions ( $n=877$, in \%).

providers' stress reports. Specifically, predictor variables were the interruption rates plus patient load and ED staffing to adjust the interruption estimates and outcome variable was work stress treated as continuous variable. Analyses were applied to data aggregated at session level. SPSS V.24.0 was used for statistical analyses (IBM).

\section{RESULTS}

Fifty $(62.5 \%)$ observation sessions were conducted with ED nurses and $30(37.5 \%)$ with ED physicians. Number and duration of observation sessions were comparable across ED treatment areas. For $\mathrm{n}=77$ observation sessions, observational data and professionals' self-evaluations were matched. Average duration of observation sessions was $91.05 \mathrm{~min}$ ( $\mathrm{SD}=4.44 \mathrm{~min}$; range $77.4-106 \mathrm{~min}$ ).

\section{Sources and contents of observed workflow interruptions}

Total observation time for the 77 sessions was 116 hours and $51 \mathrm{~min} . \mathrm{n}=877$ workflow interruptions were overall observed which resulted in an overall mean rate of 7.51 interruptions/hour. For the mean session level rates we obtained 7.53 interruptions/hour which varied across observation sessions $(\mathrm{SD}=3.9$, range $0.67-19.3$ ). Figure 1 depicts the observed frequency of observed sources: interruptions by ED colleagues of other professions were most frequent ( $\mathrm{n}=238$, mean rate/hour \pm SD 2.04 \pm 1.84 ), followed by ED colleagues of the same profession $(n=211$, $\mathrm{M}=1.81 \pm 1.72)$, and telephone/beeper-related interruptions ( $\mathrm{n}=184, \mathrm{M}=1.58 \pm 1.3$ ). All other interruption sources were recorded less frequently: interruptions by patients $(\mathrm{n}=70, \mathrm{M}=0.6 \pm 0.86)$, interruptions due to information impediments or problems $(\mathrm{n}=52, \mathrm{M}=0.45 \pm 0.72)$, interruptions due to equipment problems $(\mathrm{n}=50, \mathrm{M}=0.43 \pm 0.62)$, interruptions by patient's relatives $(\mathrm{n}=43, \mathrm{M}=0.37 \pm 0.59)$, and by any other person $(\mathrm{n}=29, \mathrm{M}=0.25 \pm 0.56)$.
Figure 2 presents the distribution of $n=791$ observed contents of interruption events. Most frequently were interruptions related to parallel cases $(n=240$, $\mathrm{M}=2.07 \pm 1.84$ ), followed by interruptions concerning the current case $(\mathrm{n}=151, \mathrm{M}=1.28 \pm 1.26)$, and communication related to coordination activities $(\mathrm{n}=144, \mathrm{M}=1.24 \pm 1.37)$. Others occurred less frequently: interruptions referring to completed cases $(\mathrm{n}=115, \mathrm{M}=0.99 \pm 1.10)$, patient comfort-related interruptions $(\mathrm{n}=34, \quad \mathrm{M}=0.30 \pm 0.62)$, time-critical information concerning a new case $(n=18$, $M=0.16 \pm 0.48)$, and other communication contents ( $n=89$, $\mathrm{M}=0.76 \pm 0.81$ ).

\section{ED professionals' ratings of interruptions and work stress}

Concomitant evaluations of observed ED professionals were obtained immediately after the observation session. Perceived interruption frequency was $\mathrm{M}=4.34$ ( $\mathrm{SD}=2.57$ ). These ratings did not differ between nurses and physicians. Professionals working in the observation and clinical decision unit $(\mathrm{M}=5.31, \mathrm{SD}=2.46)$ reported significantly more interruptions than ED professional working in the trauma section $(\mathrm{M}=3.17, \mathrm{SD}=2.59 ; \mathrm{F}(\mathrm{df}=2)=5.08$, $\mathrm{P}<0.01)$.

Perceived stress was $\mathrm{M}=10.8$ (sum score, $\mathrm{SD}=2.91$; scale range 6-24). ED professionals' work stress reports were normally distributed. There was no significant difference between professions. However, professionals in the trauma section reported lower stress levels $(\mathrm{M}=9.27$, $\mathrm{SD}=3.24)$ than professionals in the internal medicine section $(\mathrm{M}=11.37, \mathrm{SD}=2.69)$ and observation and clinical decision unit $(\mathrm{M}=11.77, \mathrm{SD}=3.12 ; \mathrm{F}(\mathrm{df}=2)=6.28, \mathrm{P}=0.03)$.

Concerning control variables, overall ED patient load was positively correlated with professionals' stress reports $(\mathrm{r}=0.31, \mathrm{P}=0.01)$. We also identified an association between ED staffing and ED professionals' stress $(r=0.26$, 


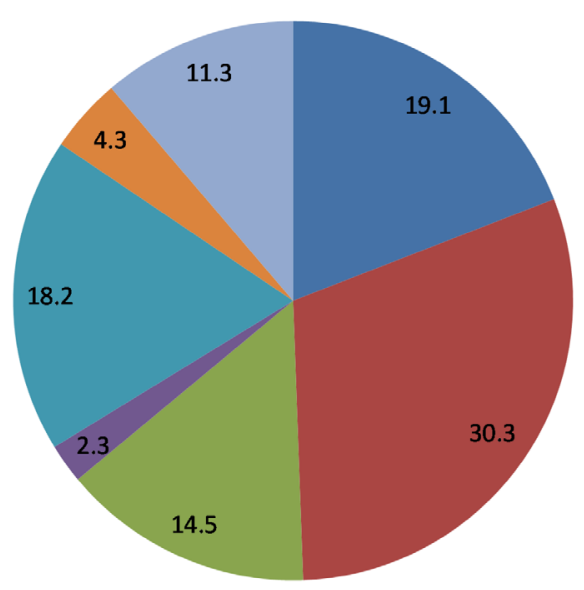

related to current case

related to parallel case

related to completed cases

related to a new case (timecritical)

related to coordination

related to patient comfort

other contents

Figure 2 Contents of emergency department (ED) providers' workflow interruptions ( $\mathrm{n}=791$, in \%).

$\mathrm{P}=0.02)$. Patient acuity was associated with patient load $(\mathrm{r}=0.48, \mathrm{P}<0.01)$ but neither related to professionals' stress reports $(\mathrm{r}=0.13, \mathrm{P}=0.26)$ nor to observed rates of interruption sources and contents.

\section{Observed interruptions and ED professionals' work stress}

After controlling for patient load and ED staffing, we found that higher rates of overall interruptions were related to increased reports of interruption frequency $(\beta=0.46, P<0.01)$. This corroborates the validity of our approach such that interruption counts by observers were related to perceptions of observed ED professionals.

Table 2 reports associations between the three most frequent sources and contents of workflow interruptions

Table 2 Associations between interruption sources, contents and emergency department (ED) providers' work stress

\begin{tabular}{|c|c|}
\hline & Outcome \\
\hline & $\begin{array}{l}\text { ED providers' } \\
\text { work stress }\end{array}$ \\
\hline Determinant & $(\beta, P)$ \\
\hline \multicolumn{2}{|l|}{ Source of workflow interruptions } \\
\hline Overall (Sum score) & $0.10,0.34$ \\
\hline ... by colleagues of the same profession & $0.08,0.50$ \\
\hline ... by colleagues of another profession & $0.05,0.68$ \\
\hline ... by telephone/beeper & $-0.01,0.97$ \\
\hline \multicolumn{2}{|l|}{ Content of workflow interruptions } \\
\hline ... related to current case under care & $0.05,0.63$ \\
\hline ... related to parallel case under care & $0.22,0.045$ \\
\hline ... related to coordination activities & $-0.03,0.76$ \\
\hline
\end{tabular}

$n=77$ observation sessions; $\beta$, regression coefficient; $P, P$ value; adjusted for patient load (number of patients registered) and ED staffing (number of ED staff present). and ED professionals' work stress. The multivariate models satisfied the linear regression assumptions.

No associations between the three most frequent interruption sources and ED professionals' work stress were identified (cf, table 2). However frequent interruptions relating to parallel cases under care contributed significantly to increased work stress among ED professionals. This association remained robust after controlling for patient load and staffing.

\section{DISCUSSION}

This mixed-methods study identified sources and contents of workflow interruptions in a multidisciplinary ED. For the majority of observed interruption events and sources, no evidence for associations with provider stress at work was found. However, interruption events referring to parallel cases under care increased work stress among ED physicians and nurses. Our findings contribute to the knowledge base on ED workflow interruptions and work stress in several ways.

Our first aim was to apply a tool for expert observations with particular focus on sources and contents of interruptions. The vast majority of interruptions were caused by ED colleagues of the same and other ED professions as well as by telephone/beepers. These three sources accounted for almost $75 \%$ of all interruptions. This finding reflects the continuous need for intraprofessional and interprofessional communication within ED teams. ${ }^{3}$ Previous research in critical care settings showed that clinicians often assign high priority to interactions with colleagues. $^{2}$

To the best of our knowledge, this is the first study that sought to quantify contents of interruptive communication events in ED workflows. We found that almost one-third of all interruptions were related to parallel cases under care. This indicates the substantial share of 
ED communication that contains prompts for different patients receiving care. ${ }^{25}$ Interruptions referring to the current case as well as to coordination activities were also prevalent. ${ }^{3}$ However, comfort-related interruptions that benefitted patients or their relatives were rare in comparison with interprofessional interruption contents. ${ }^{14}$ We also observed that interruptions containing urgent alerts occurred rarely.

Our second aim was to determine associations between workflow interruptions and ED professionals' work stress. Our approach provides a quantification of associations between interruptions and ED clinicians' stress experiences. The three most prevalent sources of workflow interruptions were not related to subsequent work stress reports after $90 \mathrm{~min}$ observation sessions. Two potential conclusions are drawn. (1) The mere count and frequency of interruptions from different sources do not contribute to ED professionals' work stress. (2) Referring to the magnitude of reported associations (in table 2), the effect of interruption rates on clinicians' stress was too small to detect within our study and its statistical power. Previous research of ED professionals showed that interruptions are perceived as disturbing or non-disturbing based on various factors of the interruption process such as content of the interruption, clinician's individual constitution and external factors. ${ }^{18}$

Nevertheless, taking into account the content of interruptions, we identified one significant association: increased interruption events on parallel cases under care were associated with increased self-reported work stress. This association was robust after controlling for patient load and ED staffing. Previous research found that interruptions were particularly detrimental during direct care activities by causing breaks in professionals' attention and patient treatment processes. ${ }^{14}$ Thus, interruption events containing information on parallel cases may divert the attentional focus of ED professionals from the current case under care, increase their work stress and eventually impair performance. Case-irrelevant information may evoke multitasking and task-switching behaviours and thus contribute to increased mental workload and work stress. ${ }^{2}$ Moreover, ED professionals might compensate by working faster after interruptions which increases perceived stress. ${ }^{13} 16$ Our findings resonate well with cognitive load theory which proposes that interruption events that stretch cognitive resources, that is, provide additional information on another patient under care, are demanding. Frequent cognitive shifts and interruptions contribute to cumulated mental load, potentially mitigating ED professionals' attention and awareness. ${ }^{26}$ Another potential underlying explanation for this finding might be that frequent information concerning parallel cases is indicative of high time pressure or insufficient case management among ED professionals. However, since we controlled for overall patient load and ED staffing, potential bias due to unmeasured workload is limited.

Furthermore, we found no evidence that interruptions related to the current case at hand were associated with work stress. Disruptive information that refers to the current case might be perceived as beneficial interruptions that add value or inform subsequent treatment activities. $^{721}$ Our findings are consistent with previous observations in operating room (OR) settings where case-relevant communication events contribute to smooth and effective team collaboration. ${ }^{23}$

\section{Limitations}

Several limitations apply to our study. First, observational measures do not entirely convey complexities of the sociotechnical ED system as well as the interruption process. ${ }^{1712}$ Our observation instrument does not distinguish between interruptive events that are clinically essential or appropriate and non-appropriate interruptions. This evaluation requires a strong clinical background of observers as well as careful consideration of the actual patient status which is almost unfeasible during on-site observations in highly dynamic ED care settings. Second, our observational tool underlies validity and reliability limitations that are inherent to observational research in dynamic ED environments. We acknowledge that inter-rater reliability was fair to good. Third, occasionally, exact assessment of communication contents was difficult, for example, particularly during rapid and short communication events concerning different cases. We also acknowledge that the content categories of our observational tool may bear overlap. Although stress among healthcare professionals contributes to adverse outcomes, we cannot infer about the potential impact of observed workflow interruptions on subsequent adverse patient outcomes, for example, medication administration errors. ${ }^{15} 2527$ Within our main analyses, only one out of seven associations achieved significance. Since we applied no adjustment for multiple testing, we acknowledge that our discussion concerning significant associations should be considered carefully. Fourth, results should not be transferred to other ED environments without further consideration. Future investigations should draw on multi-centre trials that encompass varying complexities and dynamics of ED work settings. Observation sessions were carried out during day shifts only. Work practices during night shifts may differ. We did not randomly assign observations across time of day. Post hoc, we tested if study variables differed significantly between morning or afternoon observation sessions. Although we did not obtain significant differences for interruption rates and self-reports (results can be obtained from the corresponding author), our random selection procedure did not take account of variability within the day. Due to confidentiality restrictions we did not assess personal information of observed ED providers. We may have observed some providers multiple times, thus we suggest for future studies to acknowledge this potential bias, that is, clustering of individuals, and potentially nested data structures in analyses. Fifth, our correlational analyses limit inferences concerning causality. Previous reviews showed that evidence concerning causal links between 
interruptions and errors is preliminary and deserves further studies with robust designs. ${ }^{21} 27$ We acknowledge that the distribution of our interruption events was skewed. Within our multivariate analyses we used linear regression that are considered robust if the predictor variable is distributed non-normally. ${ }^{28}$ Given the borderline significance of our results, future studies should draw on well-powered samples and apply analyses that take account of Poisson-like distribution of interruption counts. Finally, several studies emphasise that the impact of interruptions also depends on task-related factors, for example, case complexity. ${ }^{2} 718$

\section{Implications}

Findings of this mixed-methods study contribute to emerging literature concerning measures to understand interruptions and distractions in ED work systems. ${ }^{5} 729$ This study combined time-motion assessments and ED professionals' self-evaluations. Our findings advocate that the exclusive focus on interruption sources might limit inferences concerning the impact of interruptive communication on ED professionals' work stress. Taking into account the contents of interruption events may meaningfully expand the scope of previous research attempts that almost exclusively focused on sources of interruptions. ${ }^{7}$ Future research should also take account of social implications within the interruption process and investigate sequelae of disruptions beyond the individual level, for example, through social network analysis. ${ }^{30}$ Potentially, adverse or beneficial effects of interruptions on the individual level, may be outweighed on the ED team level, for example, an individually disturbing interruption may assist team workflow or mitigate larger team-level stressors. ${ }^{730}$

Concerning potential implications for ED practice, we found that interruptions including information on parallel cases are a frequent phenomenon and have an adverse effect on ED professionals' work stress. Yet, we assume that their strict prevention might implicate detrimental effects for efficiency and collaboration in ED workflows. Since interruptions in ED work unfold in a complex sociotechnical work and care environment, ${ }^{729}$ interventions to handle interruptions should consider this study's results in two ways. First, the mere count of interruption sources might be an ineffective approach to mitigate ED professionals' work stress. Future interventions in this field should focus on the contents of interruptions and actual purpose of interruptive communication. Moreover, interventions that target work stress in ED professionals should limit demands that concur with cognitive overload (ie, high working memory demands) or multitasking demands triggered through communication that does not relate to current cases. ${ }^{31}$ Work and process redesign approaches that seek to reduce disruptive communication in the ED, should consider interruption content and provider outcomes when mitigating workflow interruptions. ${ }^{26}$ Finally, our study neglected further organisational, care and safety-related outcomes that should be considered in future investigations, that is, workflow, collaboration and efficiency in care.

\section{CONCLUSIONS}

Our results highlight the importance of understanding interruptions and work stress within the ED environment. We found that the mere count of interruption sources was not associated with ED professionals' stress at work. Yet, interruptions containing information on parallel cases under care contributed to increased stress at work. Future investigations should further elucidate potential hazards and benefits of interruption events in ED work in the light of different safety and provider outcomes.

Acknowledgements We would like to thank Tobias Filmer, Jeannette Weber, Theresa Steeb and Nikolaus Bürger for their support in data collection. The results reported were part of the Doctoral Thesis requirements of Joana Beck (LudwigMaximilians-University Munich).

Contributors MWL and AS conceived the research idea. MWL, JB, MWR and AS contributed to the study design, collection and analysis of the data. MWL, JB, MWR and AS contributed to the drafts and revisions of the manuscript.

Funding This study was partly funded by the Munich Centre for Health Sciences (MC-Health).

Competing interests None declared.

Ethics approval The Ethics Committee of the Medical Faculty, Munich University, approved the study (NR 327-15).

Provenance and peer review Not commissioned; externally peer reviewed.

Data sharing statement Anonymised and deidentified data will be made available to other investigators upon request. Interested readers should contact the corresponding author MWL (matthias.weigl@med.Imu.de) to obtain the data.

Open Access This is an Open Access article distributed in accordance with the Creative Commons Attribution Non Commercial (CC BY-NC 4.0) license, which permits others to distribute, remix, adapt, build upon this work non-commercially, and license their derivative works on different terms, provided the original work is properly cited and the use is non-commercial. See: http://creativecommons.org/ licenses/by-nc/4.0/

(C) Article author(s) (or their employer(s) unless otherwise stated in the text of the article) 2017. All rights reserved. No commercial use is permitted unless otherwise expressly granted.

\section{REFERENCES}

1. Wears RL, Perry SJ. Human factors and ergonomics in the emergency department. Ann Emerg Med 2002;40:206-12.

2. Walter SR, Li L, Dunsmuir WT, et al. Managing competing demands through task-switching and multitasking: a multi-setting observational study of 200 clinicians over 1000 hours. BMJ Qual Saf 2014;23:231-41.

3. Nugus P, Holdgate A, Fry M, et al. Work pressure and patient flow management in the emergency department: findings from an ethnographic study. Acad Emerg Med 2011;18:1045-52.

4. Wears RL, Woloshynowych M, Brown R, et al. Reflective analysis of safety research in the hospital accident \& emergency departments. Appl Ergon 2010;41:695-700.

5. Chisholm CD, Collison EK, Nelson DR, et al. Emergency department workplace interruptions: are emergency physicians "interrupt-driven" and "multitasking"? Acad Emerg Med 2000;7:1239-43.

6. Weigl M, Müller A, Holland S, et al. Work conditions, mental workload and patient care quality: a multisource study in the emergency department. BMJ Qual Saf 2016;25:499-508.

7. Werner NE, Holden RJ. Interruptions in the wild: development of a sociotechnical systems model of interruptions in the emergency department through a systematic review. Appl Ergon 2015;51:244-54.

8. Ratwani RM, Fong A, Puthumana JS, et al. Emergency physician use of cognitive strategies to manage interruptions. Ann Emerg Med 2017;70:683-7. 
9. Brixey JJ, Robinson DJ, Johnson CW, et al. A concept analysis of the phenomenon interruption. ANS Adv Nurs Sci 2007;30:E26-42.

10. Chisholm CD, Weaver CS, Whenmouth $L$, et al. A task analysis of emergency physician activities in academic and community settings. Ann Emerg Med 2011;58:117-22.

11. Chisholm CD, Dornfeld AM, Nelson DR, et al. Work interrupted: a comparison of workplace interruptions in emergency departments and primary care offices. Ann Emerg Med 2001;38:146-51.

12. Berg LM, Källberg AS, Göransson KE, et al. Interruptions in emergency department work: an observational and interview study. BMJ Qual Saf 2013;22:656-63.

13. Westbrook JI, Coiera E, Dunsmuir WT, et al. The impact of interruptions on clinical task completion. Qual Saf Health Care 2010;19:284-9.

14. Myers RA, McCarthy MC, Whitlatch A, et al. Differentiating between detrimental and beneficial interruptions: a mixed-methods study. BMJ Qual Saf 2016;25:881-8.

15. Westbrook JI, Woods A, Rob Ml, et al. Association of interruptions with an increased risk and severity of medication administration errors. Arch Intern Med 2010;170:683-90.

16. Cole G, Stefanus D, Gardner H, et al. The impact of interruptions on the duration of nursing interventions: a direct observation study in an academic emergency department. BMJ Qual Saf 2016;25:457-65.

17. Jeanmonod R, Boyd M, Loewenthal M, et al. The nature of emergency department interruptions and their impact on patient satisfaction. Emerg Med J 2010;27:376-9.

18. Berg LM, Källberg AS, Ehrenberg A, et al. Factors influencing clinicians' perceptions of interruptions as disturbing or nondisturbing: a qualitative study. Int Emerg Nurs 2016;27:11-16.

19. Grundgeiger T, Dekker S, Sanderson P, et al. Obstacles to research on the effects of interruptions in healthcare. BMJ Qual Saf 2016;25:392-5.

20. Tucker AL, Spear SJ. Operational failures and interruptions in hospital nursing. Health Serv Res 2006;41:643-62.
21. Rivera-Rodriguez AJ, Karsh BT. Interruptions and distractions in healthcare: review and reappraisal. Qual Saf Health Care 2010;19:304-12.

22. Weigl M, Müller A, Vincent $\mathrm{C}$, et al. The association of workflow interruptions and hospital doctors' workload: a prospective observational study. BMJ Qual Saf 2012;21:399-407.

23. Tschan F, Seelandt JC, Keller S, et al. Impact of case-relevant and case-irrelevant communication within the surgical team on surgicalsite infection. Br J Surg 2015;102:1718-25.

24. Arora S, Hull L, Sevdalis N, et al. Factors compromising safety in surgery: stressful events in the operating room. Am J Surg 2010;199:60-5

25. Walter SR, Raban MZ, Dunsmuir WTM, et al. Emergency doctors' strategies to manage competing workload demands in an interruptive environment: an observational workflow time study. App Ergon 2017;58:454-60.

26. Potter P, Wolf $L$, Boxerman S, et al. Understanding the cognitive work of nursing in the acute care environment. $J$ Nurs Adm 2005;35:327-35.

27. Raban MZ, Walter SR, Douglas HE, et al. Measuring the relationship between interruptions, multitasking and prescribing errors in an emergency department: a study protocol. BMJ Open 2015:5:e009076.

28. Ernst AF, Albers CJ. Regression assumptions in clinical psychology research practice-a systematic review of common misconceptions. PeerJ 2017;5:e3323.

29. Morrison JB, Rudolph JW. Learning from accident and error: avoiding the hazards of workload, stress, and routine interruptions in the emergency department. Acad Emerg Med 2011;18:1246-54.

30. McCurdie T, Sanderson P, Aitken LM. Applying social network analysis to the examination of interruptions in healthcare. Appl Ergon 2018;67(Suppl C):50-60

31. Douglas HE, Raban MZ, Walter SR, et al. Improving our understanding of multi-tasking in healthcare: drawing together the cognitive psychology and healthcare literature. Appl Ergon 2017;59:45-55 